\title{
Nuclear enthalpies
}

\author{
Jacek Rozynek ${ }^{1, a}$ \\ ${ }^{1}$ National Centre for Nuclear Studies, Hoza 69, 00-781 Warsaw, Poland
}

\begin{abstract}
Even small departures from a nuclear equilibrium density with constant nucleon masses require an increase of a nucleon enthalpy. This process can be described as volume corrections to a nucleon rest energy, which are proportional to pressure and absent in a standard Relativistic Mean Field (RMF) with point-like nucleons. Bag model and RMF calculations show the modifications of nucleon mass, nucleon radius and a Parton Distribution Function (PDF) of Nuclear Matter (NM) above the saturation point originated from the pressure correction.
\end{abstract}

\section{Introduction}

The finite size correction to the nucleon rest energy connected with the nucleon volume $\Omega_{N}$ will be investigated in the compressed nuclear matter (NM) at the pressure $p$. In such a compressed medium, the nucleon constituents - quarks and gluons have to do an additional work $W_{N}=p \Omega_{N}$ to keep a space $\Omega_{N}$ for a nucleon bags". The nucleon mass is a result of strong interaction between almost massless quarks and gluons. The nuclear Drell-Yan experiments [1, 2], which measure the sea quark enhancement, we described [3] with a small $1 \%$ admixture of nuclear pions and the $M_{N}$ unchanged. Thus the deep inelastic phenomenology indicates that a change of the nucleon invariant mass at the saturation density in comparison to the value in vacuum is rather negligible although nuclear scalar and vector mean fields are strong [4]. Therefore, when the energy transfer from Nucleon-Nucleon (NN) interaction to the partons is absent the nucleon mass should decrease with pressure. It will involve functional corrections to a nucleon rest energy, dependent from external pressure with a physical parameter - a nucleon radius $R_{0}$.

Alternatively, at stronger $\mathrm{NN}$ repulsion we should consider the possible energy transfer from NN interactions to the confined space inside nucleon, maintaining the constant mass of the nucleon for higher densities. Other modifications connected with finite volume of nucleons, like correlations of their volumes, will be neglected. In order to discussed this issue we introduce a nucleon enthalpy $H_{N}$ in NM with a density $\varrho\left(\varrho_{0}\right.$ at equilibrium) and "external" nuclear pressure $p$

$$
H_{N}(\varrho) \doteq M_{p r}(\varrho)+p \Omega_{N} \text { with } H_{N}\left(\varrho_{0}\right)=M_{N},
$$

as a "useful" expression for the total rest energy of a immersed nucleon "bag". The nucleon mass $M_{p r}$ is possibly modified in the compressed medium. For the constant nucleon mass $M_{p r}$ in NM, the formula (1) shows that the enthalpy $H_{N}$ - as the total nucleon rest energy - increases. In such a

\footnotetext{
a e-mail: rozynek@fuw.edu.pl
} 
case a part of nucleon-nucleon interaction energy is transferred to the extended system of strongly interacting partons.

Let us introduce analogously the single particle nuclear enthalpy

$$
H_{A}^{T} / A=\varepsilon_{A}+p / \varrho=E_{F}
$$

where $\varepsilon_{A}=E_{A} / A$ is a single nucleon energy and $E_{F}$ is the nucleon Fermi energy $E_{F}$ or chemical potential $\mu$. In order to compare energy densities let us move to the specific enthalpies which are given respectively by:

$$
\begin{aligned}
h_{A}^{T}(\varrho) \doteq \frac{H_{A}^{T}}{E_{A}} & =1+\frac{p}{\varrho \varepsilon_{A}(\varrho)} \\
h_{N}(\varrho) \doteq \frac{H_{N}}{M_{p r}} & =1+\frac{p}{\varrho_{c p}(\varrho) M_{p r}(\varrho)}
\end{aligned}
$$

where $\varrho_{c p}=1 / \Omega_{N}$ is a close packing density for extended nucleons inside NM. The specific nuclear enthalpy $h_{A}^{T}\left(\varrho_{0}\right)$ in equilibrium density $\varrho_{0}$ is smaller than the specific nucleon enthalpy $h_{N}\left(\varrho_{0}\right)$ but increases faster than the nucleon one. It is easy to show that the equality of these specific enthalpies at a certain density $\varrho_{c r}$

$$
h_{A}^{T}\left(\varrho_{c r}\right)=h_{N}\left(\varrho_{c r}\right)
$$

is equivalent to a following condition for the critical density $\varrho_{c r}$

$$
\varrho_{c r} \varepsilon_{A}\left(\varrho_{c r}\right)=\varrho_{c p}\left(\varrho_{c r}\right) M_{p r}\left(\varrho_{c r}\right) .
$$

where the alignment of energy densities, outside and inside nucleon, takes place. Another word, energy density $\left(\varrho \varepsilon_{A}\right)$, which includes a space $\Omega_{A-}$ between nucleons, reaches the energy density of a quark plasma $\left(\varrho_{c p} M_{p r}\right)$ inside nucleon therefore an ultimate de-confinement transition to the QuarkGluon-Plasma (QGP) will take place when condition (5) or (4) is satisfied.

\section{The nucleon rest energy in the Bag model in NM}

Let us discuss the relation (1) in the simple bag model where the nucleon in the lowest state of three quarks is a sphere of a volume $\Omega_{N}$. In a compressed medium [5], pressure generated by free quarks inside the bag [6] is balanced at the bag surface not only by intrinsic confining "pressure" $B(\varrho)$ but also by nuclear pressure $p$; generated e.q. by elastic collisions with other hadron $[7,8]$ bags, also derived in QMC model in a medium [9]. A mass $M_{p r}$ for finite $p(\varrho)$ can be obtained like a general expresion[6, 11] on a bag energy in a vacuum $E_{B a g}^{0}\left(R_{0}\right) \propto 1 / R_{0}$ as a function of the radius $R_{0}$ with phenomenological constants - $\omega_{0}, Z_{0}[9]$ and a bag "constant" $B(\varrho)$. But now, in equilibrium internal parton pressure $p_{B}$ inside the bag is equal (cf. [9]), on a bag surface, nuclear pressure $p$

$$
p=p_{B}=\frac{3 \omega_{0}-Z_{0}}{4 \pi R^{4}}-B(\varrho) \rightarrow(B(\varrho)+p) R^{4}=\text { const }
$$

and we get the nucleon radius depending from $B+p_{H}$ :

$$
R(\varrho)=\left[\frac{3 \omega_{0}-Z_{0}}{4 \pi(B(\varrho)+p(\varrho))}\right]^{1 / 4} .
$$

Thus, the pressure $p(\varrho)$ between the hadrons acts on the bag surface similarly to the bag "constant" 

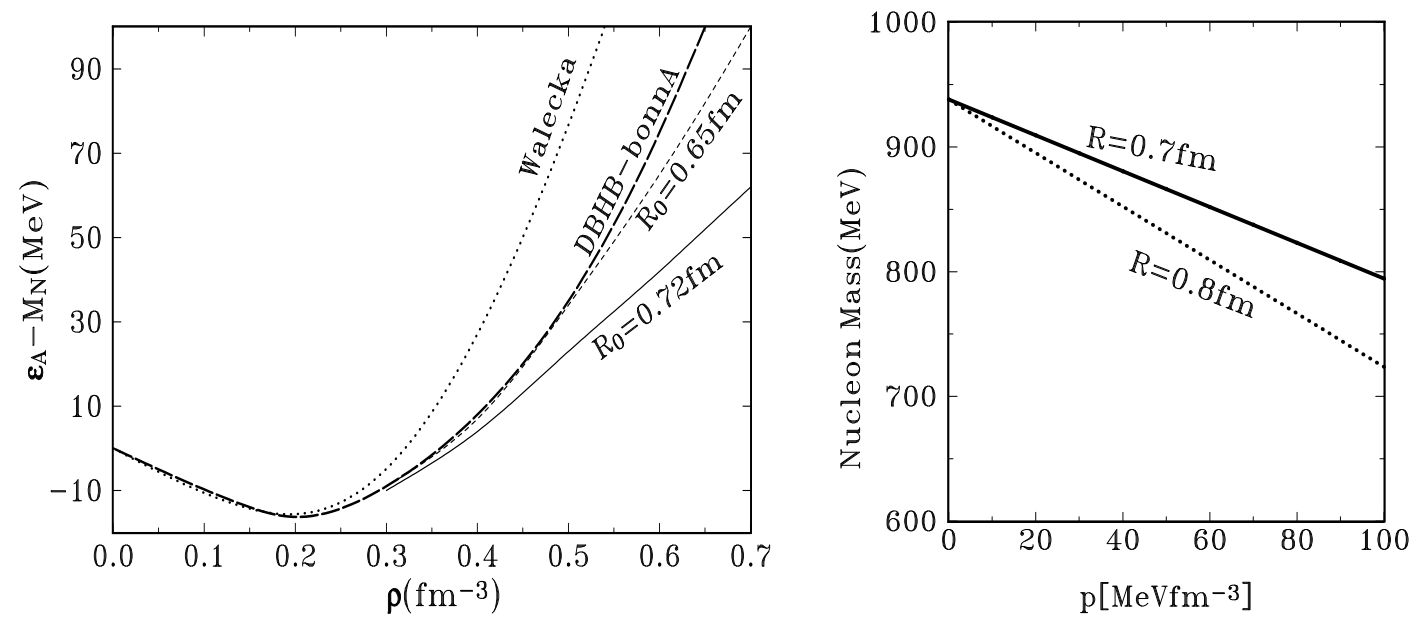

Figure 1. Left panel - energy of NM above the equilibrium density for different $[4,11,12]$ models. Right panel - the nucleon mass $M_{p r}$ as a function of NM pressure for two constant nucleon radii R=0.7,0.8.
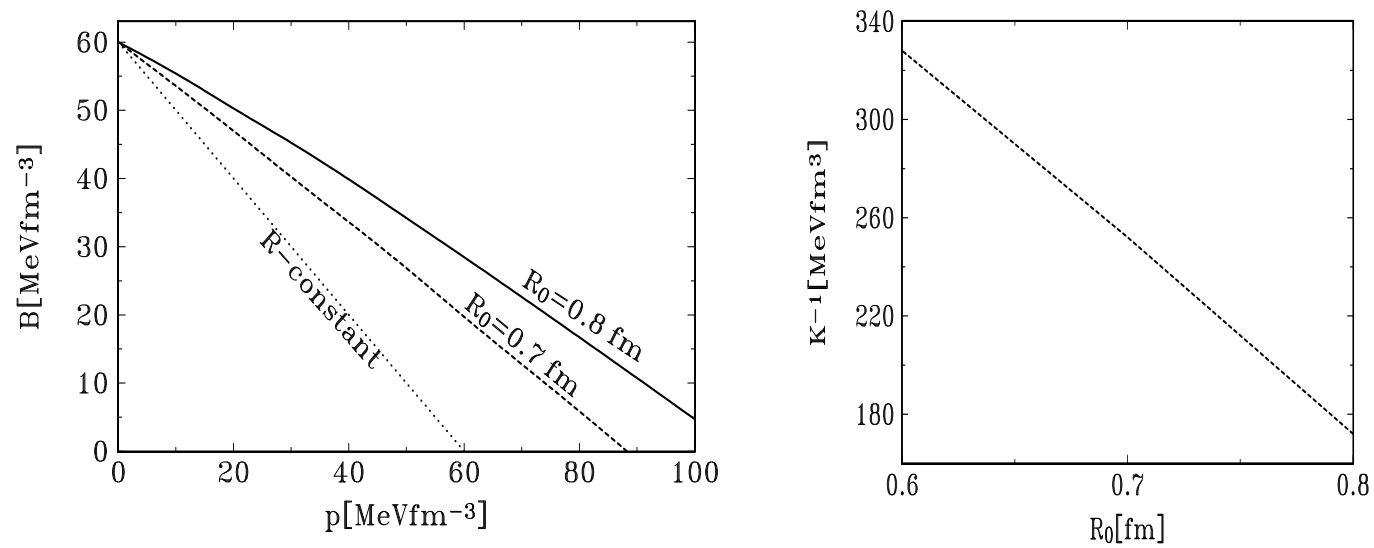

Figure 2. Left panel - pressure dependent bag "constant" $B$ for the given nucleon radius $R=0.7 \mathrm{fm}$ (case A constant nucleon size) and for two different values of $R_{0}=0.7,0.8 \mathrm{fm}$ (case B - constant nucleon mass). Right panel - the nuclear compressibility as a function of nucleon constant radius. $\mathrm{R}=0.7,0.8 \mathrm{fm}$ (case $\mathrm{B}$ ). 
$B(\varrho)$. Consequently the mass in NM

$$
M_{p r}(\varrho)=\frac{4}{3} \pi R^{3}[4(B+p)-p]=E_{B a g}^{0} \frac{R_{0}}{R}-p \Omega_{N}
$$

The density dependent nucleon mass is shown in Fig.1 (right panel) for the constant radius $R$. The scaling factor $R_{0} / R$ comes from a well-known model dependence $\left(E_{b a g}^{0} \propto 1 / R_{0}\right)$ in the spherical bag [6]. This simple radial dependence is now lost in (7) and responsible for that is the pressure dependent correction to the mass of a nucleon given by the product $p \Omega_{N}$. This term is identical with the work $W_{N}$ in (1) and disappear for the nucleon enthalpy

$$
H_{N}(\varrho)=M_{p r}+p \Omega_{N}=E_{B a g}^{0} \frac{R_{0}}{R(\varrho)} \propto 1 / R(\varrho)
$$

Thus the total rest energy of the nucleon in the nuclear medium depends only from its size $R(\varrho)$. This simple dependence from $R(\varrho)$ reflects a scale of a confinement of partons inside compressed NM. The internal pressure $B(\varrho)$ depends on the external pressure $p$ and the nucleon radius (6)

$$
B=B\left(\varrho_{0}\right)\left(R_{0} / R\right)^{4}-p .
$$

and is shown in Fig. 2 for the constant radius and the constant mass with a variable radius. Presented dependence of the bag "constant" $B$ from the nuclear density has a strong influence [10] on EoS. The Equation of State (EoS) calculated in the linear scalar-vector RMF including finite nucleon sizes [11], and presented in Fig. 1 in a comparison with other models, is softer for bigger nucleon radii $R_{0}$ with good value [13] for a compressibility $K^{-1} \sim 235 \mathrm{MeV} \mathrm{fm}^{-3}$ for $R_{0}=0.72 \mathrm{fm}$; see Fig. 1 - left panel. For a constant $R_{0}$, the nonlinear term [14] in a scalar potential is not needed, which shows that finite nucleon sizes can provide independently a correct nuclear stiffness of EoS like in DBHF calculations [12] shown in Fig.1. The difference between compressibility $K_{\Omega}^{-1}$ - with and $K_{A}^{-1}$-without volume correction to energy $\varepsilon$ can be written as:

$$
\begin{aligned}
K_{A}^{-1} & -K_{\Omega}^{-1}=9 \varrho^{2} \frac{d^{2}}{d \varrho^{2}}\left(p_{H} \Omega_{N}\right)=9 \varrho^{2} \frac{d^{2}}{d \varrho^{2}}\left[\frac{\varrho^{2} \Omega_{N}}{1-\varrho \Omega_{N}} \frac{d \varepsilon_{\Omega}}{d \varrho}\right]= \\
& =\frac{9 \varrho^{3} \Omega_{N}}{1-\varrho \Omega_{N}}\left[f(\varrho) \frac{d \varepsilon_{\Omega}}{d \varrho}+\left(\frac{6-5 \varrho \Omega_{N}}{1-\varrho \Omega_{N}}\right) \frac{d^{2} \varepsilon_{\Omega}}{d \varrho^{2}}+\varrho \frac{d^{3} \varepsilon_{\Omega}}{d \varrho^{3}}\right]
\end{aligned}
$$

where $f(\varrho)$ is an unknown regular function at the equilibrium density which multiplies the first derivative $\varepsilon^{\prime}(\varrho)$ vanishing at the $\varrho_{0}$. A last term in (10) with the third derivative is in the next order of density, also the third derivative is small if $\varepsilon_{\Omega}$ can be approximated by quadratic function of density at the vicinity of NM equilibrium. Consequently, keeping only the term with the second derivative $\varepsilon^{\prime \prime}(\varrho)$ in the second line of last expression, which is proportional to $K_{\Omega}^{-1}$, we finally obtain:

$$
K_{\Omega}^{-1} \cong\left[\frac{\left(1-\varrho \Omega_{N}\right)^{2}}{1+4 \varrho \Omega_{N}\left(1-\varrho \Omega_{N}\right)}\right] K_{A}^{-1} \simeq \frac{1}{2} K_{A}^{-1}
$$

that the compressibility $K_{\Omega}^{-1}$ which includes the volume corrections is smaller by the density dependent factor shown above in (11). The typical saturation density with $\Omega_{N} \approx 1$ provide a small $\varrho \Omega_{N} \approx 1 / 6$ for $R_{0}=0.6 \mathrm{fm}$. Consequently $K_{\Omega}^{-1} \approx K_{A}^{-1} / 2$ (inside the uniform NM). The $K_{\Omega}^{-1}$ as a function of nucleon radius is presented in the right panel of the Fig.2. Its linear behavior is consistent with formula (11). Concluding, the significant role of the nucleon finite size in nuclear properties has been shown.

This work is supported by a National Science Center of Poland, a grant DEC2013/09/B/ST2/02897. 


\section{References}

[1] D.M. Alde et al., Phys. Rev. Lett. 64, 2479 (1990).

[2] J. R. Smith and G. A. Miller, Phys. Rev. C 65, 015211,055206 (2002).

[3] J. Rożynek, Nucl. Phys. A 755, 357c (2004).

[4] B. D. Serot and J. D. Walecka, Adv. Nucl. Phys. Vol. 16 (Plenum, N. Y. 1986); R. J. Furnstahl and B. D. Serot, Phys. Rev. C 41, 262 (1990).

[5] Buballa M., Nucl. Phys. A611, 393, (1996).

[6] K. Johnson, Acta Phys. Pol. B6, 865 (1975), A. Chodos et al., Phys. Rev. D 9, 3471 (1974);

[7] L. Ferroni and V. Koch, Phys. Rev. C 79, 034905 (2009).

[8] J.I. Kapusta and Ch. Gale, "Finite Teperatures Field Theory", Cambrdge Uniwersity Press, New York 2006.

[9] Guo Hua, J. Phys. G25, 1701 (1999).

[10] Y. Liu, D. Gao, H. Guo, Nucl. Phys. A695, 353 (2001); R. T. Cahil, C. D. Roberts J. Praschifka, Ann. Phys. (NY), 188 (1988).

[11] J. Rozynek, arXiv:1406.3832,1311.3591.

[12] T. Gross-Boelting, C. Fuchs, A. Faessler, Nuclear Physics A 648, 105 (1999); E. N. E. van Dalen, C.Fuchs, A. Faessler, Phys. Rev. Lett. 95, 022302 (2005); Fuchs J. Phys. G 35, 014049 (2008).

[13] Uchida M et al. 2004 Phys. Rev. C 69 051301(R).

[14] J.Boguta, Phys. Lett. 106B, 255, (1981); N.K. Glendenning, "Compact Stars", Springer-Verlag, New York,2000. 